\title{
Non-exponential decay of base-pair opening fluctuations in DNA
}

\author{
G. Kalosakas, ${ }^{1}$ K. Ø. Rasmussen, ${ }^{2}$ and A. R. Bishop ${ }^{2}$ \\ ${ }^{1}$ Max Planck Institute for the Physics of Complex Systems, \\ Nöthnitzer Str. 38, D-01187 Dresden, Germany \\ ${ }^{2}$ Theoretical Division and Center for Nonlinear Studies, \\ Los Alamos National Laboratory, Los Alamos, New Mexico 87545
}

\begin{abstract}
We report non-exponential decay of the time-dependent autocorrelation functions of base-pair opening dynamics in a model of DNA. Complex fluctuations occur in a wide temperature range, extending from below $200 \mathrm{~K}$ up to the premelting transition regime above physiological temperatures. The observed slow fluctuations, attributed to vibrational hot-spots, are well described by a stretched exponential functional form, in the picoseconds to nanoseconds range. The temperature dependence of the corresponding relaxation rates and stretch exponents is presented and activation energies around $4-6 \mathrm{~kJ} / \mathrm{mol}$ are calculated.
\end{abstract}

PACS numbers: 87.15.Ya, 87.15.Aa, 87.14.Gg, 87.15.He

Non-exponential relaxation is a hallmark of complex behavior, and is prototypically observed in glass forming systems. There, slow relaxation, arising from complex energy landscapes, is usually described by a stretched exponential function [1-3]. Such behavior may result from inhomogeneities, leading to a distribution of relaxation times, or from cooperative molecular motions giving rise to non-trivial relaxation even locally [1].

Biomolecules certainly belong in the category of complex materials, possessing in addition, important functionalities. Complex dynamics resulting in nonexponential relaxation, spanning many decades of time, is well established in proteins [4-9]. Such observations have lead to the fruitful concepts of conformational substates and hierarchically organized energy landscapes [10].

Similarly, for the molecule containing the genetic code, non-exponential relaxation has been observed experimentally in synthetically tagged double stranded DNA. In particular logarithmic relaxation of local structural dynamics, extending from picosecond to nanosecond time scales, has been documented by time-resolved Stokes shift techniques [11]. Although such measurements can identify these motions as arising from localized dynamics, occurring at the single nucleotide level, they cannot provide detailed information on the particular degree of freedom responsible for the probed relaxation. On longer time scales, fluorescence correlation spectroscopy, having a limited time resolution, has shown evidence of multistate slow relaxation of large bubbles, representing coherent motion of many base-pairs [12].

Here we consider conformational fluctuations of Bform DNA at a single base-pair level and show, through the time dependence of local autocorrelation functions, that complex non-exponential dynamics up to nanosecond time scales occur. Homopolymer DNA sequences, poly $(\mathrm{dG})$-poly $(\mathrm{dC})$ and poly $(\mathrm{dA})$-poly $(\mathrm{dT})$, are examined, in order to isolate effects of intramolecular anharmonic interactions from those due to sequence heterogeneity.
The autocorrelation functions are calculated using the Peyrard-Bishop-Dauxois (PBD) dynamical model [13] for the base-pair stretchings of double stranded DNA. The success of this model in describing base-pair openings of double-stranded DNA has been demonstrated by direct comparison with various experiments on melting transition [14], S1 nuclease cleavage [15], and denaturation bubbles [16]. The relatively simple character of the model, which isolates the relevant base-pair degrees of freedom and appropriately describes their nonlinear interactions, renders it attractive for investigating physical properties $[17,18]$ and possible functional importance [19] of large openings in DNA.

The multiple time scales of DNA dynamics are of course complicated given the complexity of the molecule and its water environment. This leads to important open questions regarding, e.g., various degrees of slaving of modes [20]. The PBD model simplifies much of this potential complexity and focuses on effective stacking constraint interactions between base-pairs, leading to specific nonlinear and entropic effects. It is very important to determine multiscale (stretched exponential) behavior inherent in these effects, so that they can be isolated from other timescales which may appear in even more microscopic descriptions of the molecule and its environments. Our study is an important step in this direction.

In the PBD description, the evolution of the stretching $y_{n}$ of the base-pair at site $n$ of the DNA sequence is given by

$$
m \frac{d^{2} y_{n}}{d t^{2}}=-V^{\prime}\left(y_{n}\right)-W^{\prime}\left(y_{n-1}, y_{n}\right)-W^{\prime}\left(y_{n}, y_{n+1}\right)
$$

where $V(y)$ is the on-site Morse potential

$$
V(y)=D\left(e^{-a y}-1\right)^{2},
$$

$W(x, y)$ represents the stacking interaction,

$$
W(x, y)=\frac{k}{2}\left(1+\rho e^{-\beta(x+y)}\right)(x-y)^{2},
$$


$m$ is the nucleotide mass, and prime denotes derivative with respect to $y_{n}$. The parameter values of the potentials are [14]: $k=0.025 \mathrm{eV} / A^{2}, \rho=2, \beta=0.35 A^{-1}$ for the stacking interaction, while for the Morse potential $D_{G C}=0.075 \mathrm{eV}, a_{G C}=6.9 A^{-1}$ for GC base-pairs, and $D_{A T}=0.05 \mathrm{eV}, a_{A T}=4.2 A^{-1}$ for AT pairs. The mass $m$ equals 300 amu. In our simulations reported below we consider the case of homopolymer DNA, for both kinds of base-pairs, with a length of $N=100$ sites and periodic boundary conditions, appropriate for describing long homogeneous sequences.

Solving the equations of motion (1) at a fixed energy $E$ (which is conserved during the evolution) for a large number of different realizations, we calculate the time dependence of two kinds of autocorrelation functions: the local displacement autocorrelation function

$$
C_{D}(t)=\left\langle y_{n}(t) y_{n}(0)\right\rangle-\left\langle y_{n}\right\rangle^{2}
$$

and the local energy autocorrelation function

$$
C_{E}(t)=\left\langle E_{n}(t) E_{n}(0)\right\rangle-\left\langle E_{n}\right\rangle^{2},
$$

where angle brackets denote both ensemble averages and averages along the base-pairs of the homopolymer DNA. In $\mathrm{Eq}(5), E_{n}=V\left(y_{n}\right)+\frac{1}{2} W\left(y_{n-1}, y_{n}\right)+\frac{1}{2} W\left(y_{n}, y_{n+1}\right)$ is the amount of energy localized at the base-pair $n$. The total energy is $E=\sum_{n} E_{n}$, where the sum extends over the $N$ base-pairs of the sequence. Note that the correlation functions (4) and (5) are not normalized to unity.

Working in the microcanonical ensemble, the temperature $T$ corresponding to a fixed value of $E$ is obtained through the mean kinetic energy $\left\langle E_{n}^{k i n}\right\rangle=\left\langle\frac{m}{2}\left(\frac{d y_{n}}{d t}\right)^{2}\right\rangle$ of a base-pair, as $T=2\left\langle E_{n}^{k i n}\right\rangle / k_{B}$, where $k_{B}$ is the Boltzmann constant. The model of Eqs. (1)-(3) exhibits different behavior above 50-150K, as compared with that of lower temperatures. This can be seen by the motion of a charge interacting with the displacements $y_{n}[21,22]$ and the position of the dominant peak in the dynamic structure factor [23]. Here we focus on the high temperature regime, which includes the biologically relevant region.

For each value of energy (temperature), one thousand different realizations are used for the calculation of $C_{D}(t)$ and $C_{E}(t)$. In each realization, at fixed energy $E$, the system of $N$ base-pairs initially starts with a random Gaussian distribution of velocities and displacements and is then equilibrated for 2 nsec. After that time we measure the correlation functions, which are assumed to represent fluctuations around equilibrium, up to a few nanoseconds.

Figure 1 shows results for the correlation function $C_{D}(t)$ at different temperatures (solid lines), for $\operatorname{poly}(\mathrm{dG})-\operatorname{poly}(\mathrm{dC})$ (upper plot) and poly $(\mathrm{dA})-\operatorname{poly}(\mathrm{dT})$ (lower plot). Both cases exhibit complex dynamics. At least two distinct relaxation processes are apparent. There are fast fluctuations, relaxing at subpicosecond
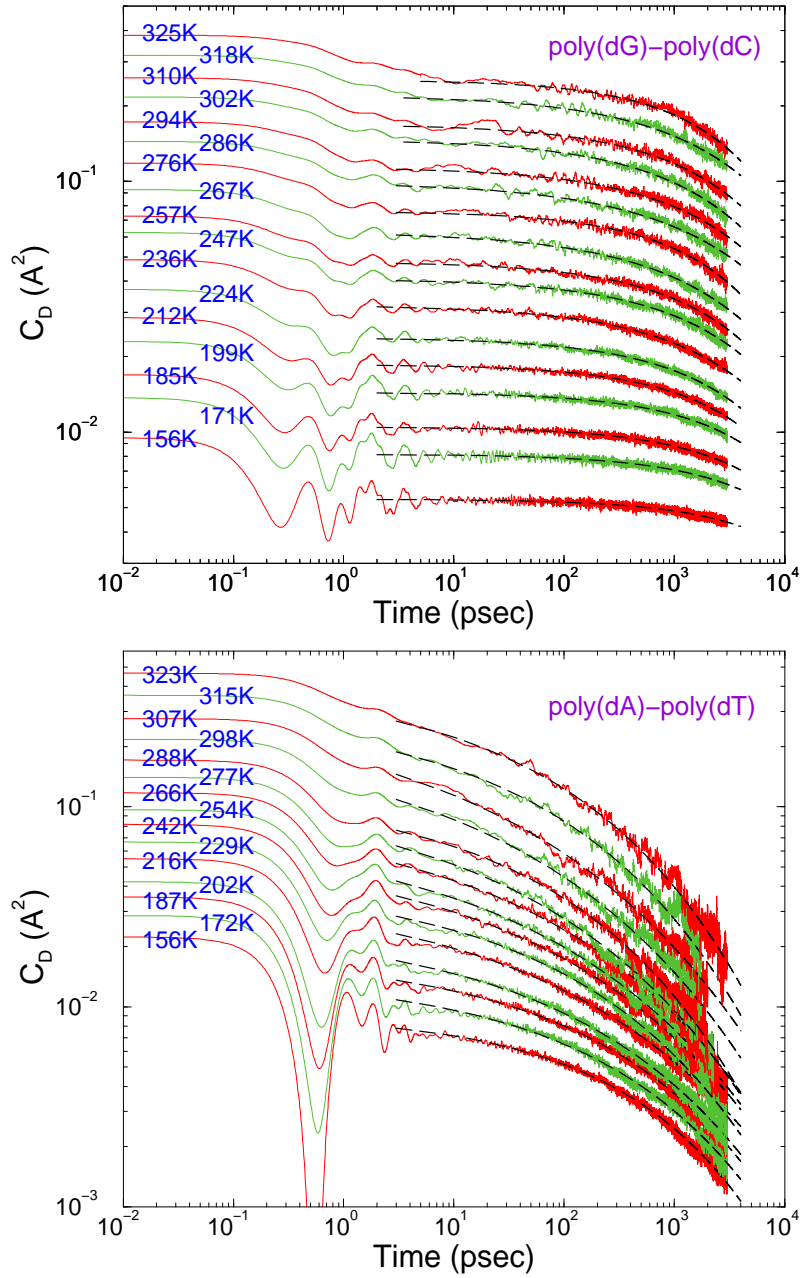

FIG. 1: Time dependence of the local displacement autocorrelation function $C_{D}(t)$, Eq. (4), for poly (dG)-poly(dC) (top) and poly (dA)-poly(dT) (bottom) DNA, at various temperatures from $150 \mathrm{~K}$ to $330 \mathrm{~K}$. Dashed lines show fits of the longtime behavior with the stretched exponential formula, Eq. (6).

time scales, followed by oscillations which are more distinct at lower temperatures. Then there is a slower relaxation extending from picoseconds up to the maximum time investigated, i.e. a few nanoseconds.

Complex fluctuations revealing fast and slower processes are also observed in the correlation function $C_{E}(t)$, shown in Fig. 2 with solid lines at various temperatures. In this case a third relaxation process is apparent at picosecond time scales, which is clearer at higher temperatures. In general the $C_{E}(t)$ curves are smoother and less noisy than the corresponding $C_{D}(t)$ ones.

In all cases the slow fluctuations are well-described by a stretched exponential dependence

$$
C(t)=C_{0} \exp \left[-\left(k_{s} t\right)^{\beta}\right] .
$$

The resulting fit of Eq. (6) to the slower relaxation of $C(t)$ is plotted in Figs. 1 and 2 with dashed lines. From this 

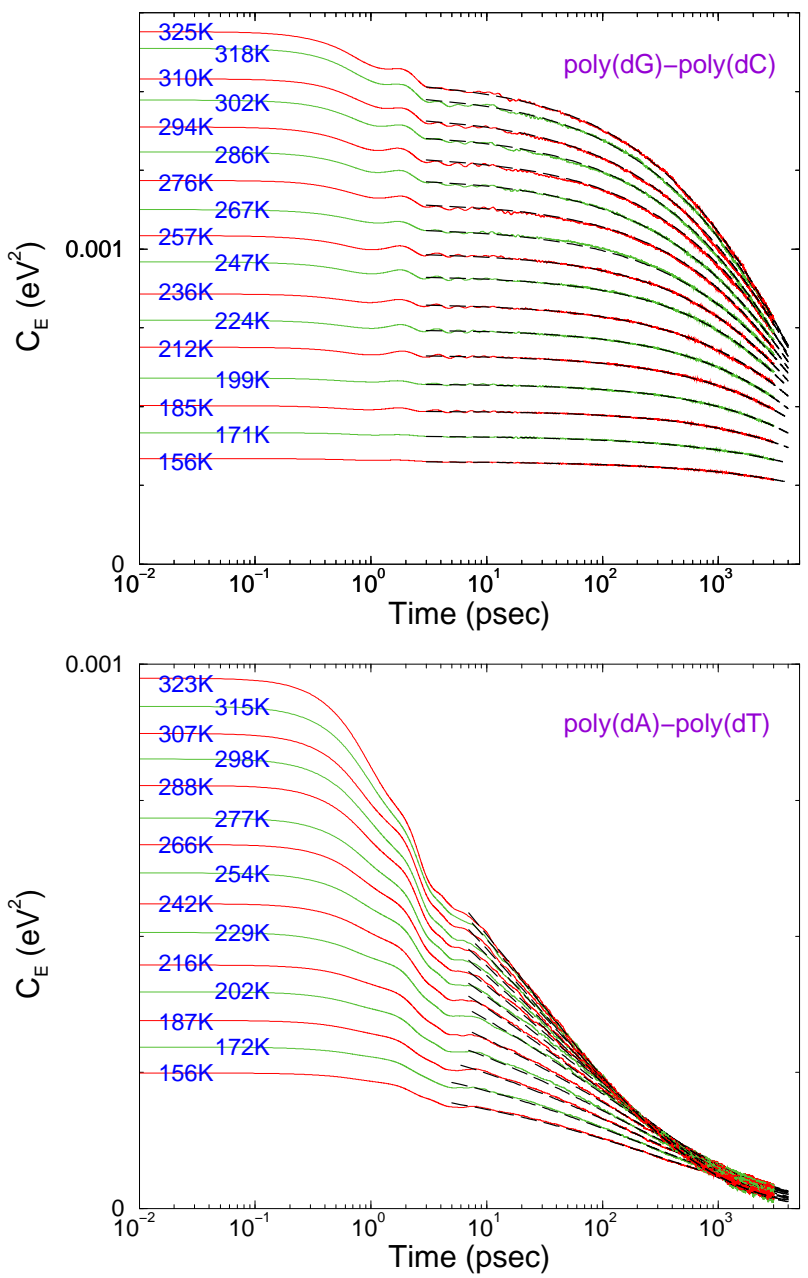

FIG. 2: Time dependence of the local energy autocorrelation function $C_{E}(t)$, Eq. (5), for poly(dG)-poly(dC) (top) and poly(dA)-poly(dT) (bottom) DNA, at various temperatures from $150 \mathrm{~K}$ to $330 \mathrm{~K}$. Dashed lines show fits of the long-time behavior with the stretched exponential form Eq. (6).

fitting the stretched exponential parameters are obtained at different temperatures.

Figure 3 depicts the temperature dependence of the stretched exponential parameters $\beta$ (upper plot) and $k_{s}$ (lower plot). These parameters are similar for the two correlation functions $C_{D}(t)$ and $C_{E}(t)$, which probe the same local fluctuations. The exponent $\beta$ is relatively insensitive to temperature changes - the obtained variations are within the errors of the fits. Temperature independence of $\beta$ is a rather common observation in glasses [1]. For poly $(\mathrm{dG})$-poly $(\mathrm{dC}), \beta$ is between 0.5 and 0.6 , while it has a significantly lower value, around 0.3 , in the case of poly $(\mathrm{dA})-\operatorname{poly}(\mathrm{dT})$. This sequence dependence is also pronounced in the rate $k_{s}$ of the stretched exponential. For poly $(\mathrm{dA})-\operatorname{poly}(\mathrm{dT})$ it is two orders of magnitude larger than that for poly $(\mathrm{d} G)$-poly $(\mathrm{dC})$. Evidently there are quite significant differences in the complexity of the dynamics of the two sequences, and it is therefore ex-
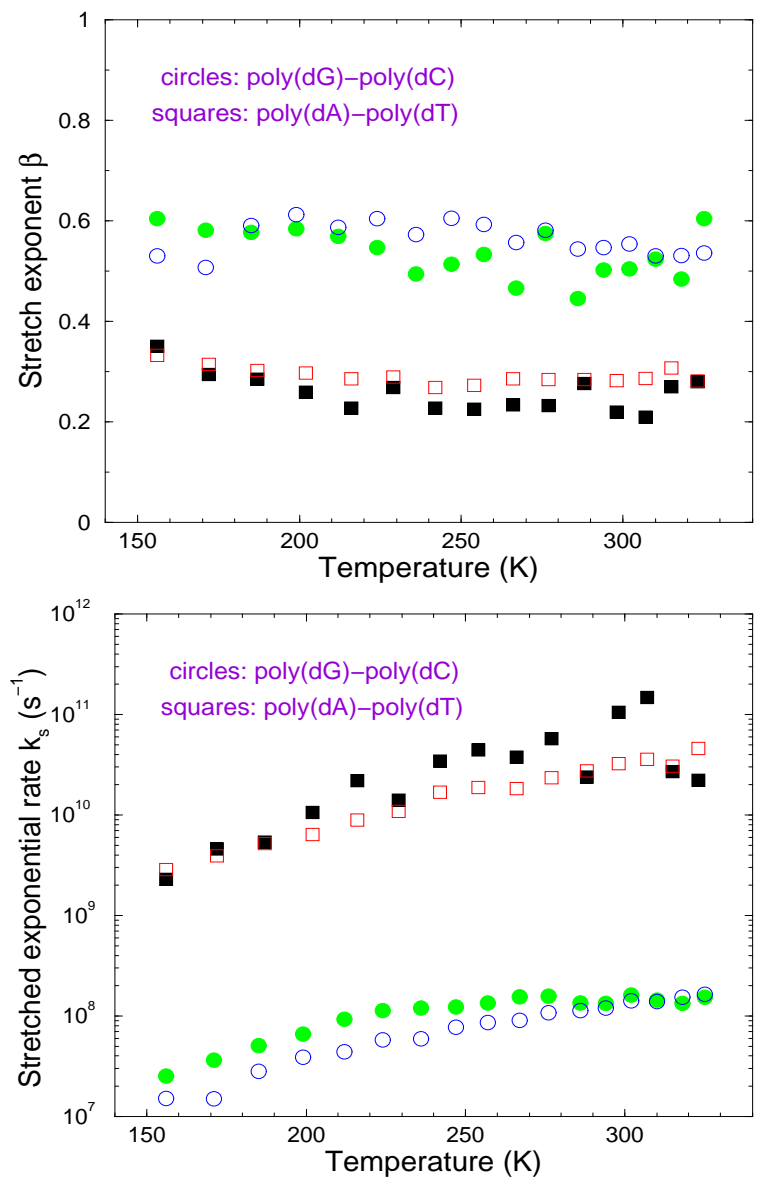

FIG. 3: Stretch exponent $\beta$ (top) and rate $k_{s}$ (bottom) of Eq. (6) for the slow fluctuations as a function of temperature for poly(dG)-poly(dC) (circles) and poly(dA)-poly(dT) (squares) DNA. Filled symbols correspond to the local displacement autocorrelation functions $C_{D}(t)$ and open to the local energy autocorrelation functions $C_{E}(t)$.

pected that in a real sequence there will be identifiable dynamical differences in the behavior of GC rich and AT rich regions.

In a relaxation of the form Eq (6), the average relaxation time $\tau_{a v}$ and the corresponding rate $k_{a v}=1 / \tau_{a v}$ can be obtained through the integral of the stretched exponential function [24-26]. This yields

$$
k_{a v}=\frac{\beta}{\Gamma(1 / \beta)} k_{s},
$$

where $\Gamma(1 / \beta)$ is the gamma function with argument $1 / \beta$. The smaller the exponent $\beta$, the smaller the average rate, as compared to $k_{s}$.

Figure 4 shows an Arrhenius plot of the average rate $k_{a v}$, obtained through Eq. (7) and the data presented in Fig. 3. Fitting the results with an Arrhenius relation

$$
k_{a v}=A \exp \left(-\frac{E_{A}}{k_{B} T}\right)
$$




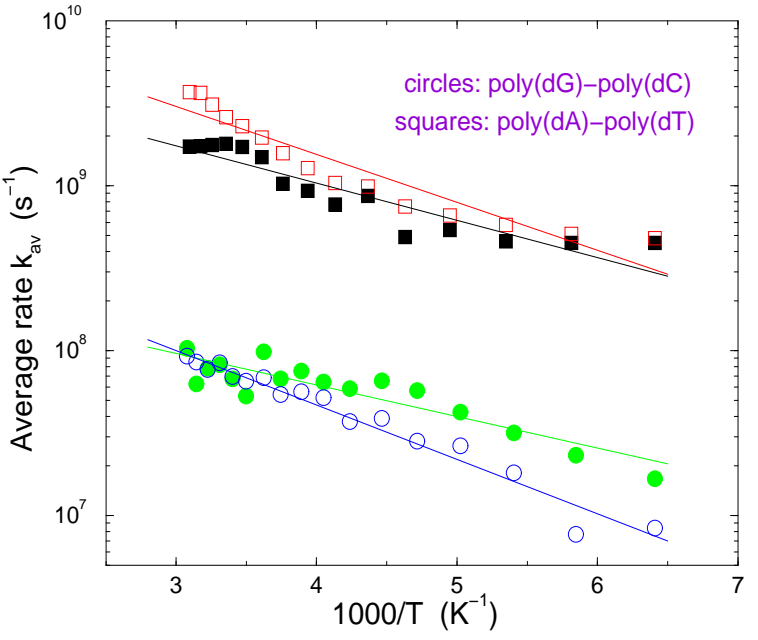

FIG. 4: Average rate $k_{a v}$, Eq. (7), for the slow fluctuations as a function of inverse temperature for poly $(\mathrm{dG})$-poly $(\mathrm{dC})$ (circles) and poly(dA)-poly(dT) (squares) DNA. Filled symbols correspond to the local displacement autocorrelation functions $C_{D}(t)$ and open to the local energy autocorrelation functions $C_{E}(t)$. Lines show fits of the corresponding data with an Arrhenius form.

we obtain an activation energy of approximately 4 $\mathrm{kJ} / \mathrm{mol}$ from the $C_{D}(t)$ data and $6 \mathrm{~kJ} / \mathrm{mol}$ from the $C_{E}(t)$ data, for both homopolymers. The fits, shown by lines in Fig. 4, are not so precise, except for the $C_{E}$ data in the poly $(\mathrm{dG})$-poly $(\mathrm{dC})$ case. Therefore, these values represent rough estimates only. The corresponding characteristic times $\tau_{a v}$ at $300 \mathrm{~K}$ are about $0.5 \mathrm{nsec}$ and 10 nsec for poly (dA)-poly(dT) and poly(dG)-poly (dC), respectively. Such time scales are consistent with the life-times of nonlinearity induced localized modes found in a simpler model with harmonic stacking interaction [27]. This suggests that vibrational hot-spots (bubbles) $[27],[21]$ are responsible for the slower, stretched exponential fluctuations presented here.

${ }^{31} \mathrm{P}$ and ${ }^{1} H$ NMR measurements [28] at room temperature have indicated the existence of local internal motions of DNA at time scales of the order of $\sim 1$ nsec. All-atom molecular dynamics simulations have shown that internal motions of DNA on this time scale are related to the rigid-body translation of subunits (bases, sugar, or phosphate groups), since the internal deformation and the rotational autocorrelation functions of rigid subunits decay faster than $10 \mathrm{psec}$ [29]. Only the rigid translations, such as the motions described by Eq. (1), have correlation times longer than 0.1 nsec. More direct observation of the motions investigated here is provided by NMR experiments of imino-proton exchange, determining open base-pair lifetimes of the order of 1-10 nsec [30]. If the probed open base-pair state is not very far from the equilibrium state considered here, then these times can be compared with the model's correlation times discussed above.

In summary, we used the simple PBD model to investigate the equilibrium fluctuations of DNA openings at a single base-pair level. Analysis of the autocorrelation functions demonstrate complex non-exponential decay with distinct glass-like behavior consisting of fast and slower processes over an extended temperature regime. The slow relaxation has been analyzed in detail and characteristic rates, stretch exponents, and activation energies are obtained. Our results suggest the need for further experimental scrutiny of DNA dynamics with a view towards possible biological roles of the long correlation times observed here for the base-pair openings. We find that the details of the ensuing dynamics, as characterized by the stretched exponential parameters $\beta$ and $k_{s}$ are very sensitive to the sequence. This clearly indicates the existence of careful structural control of the local thermal dynamics along the DNA molecule, lending support to the concept that biological action at the molecular level must be discussed in terms of dynamics [31-33], rather than in purely traditional terms of static structures.

We thank H. Frauenfelder for valuable discussions and suggestions and K. Chrissopoulou for discussions about the average rate. Research at Los Alamos National Laboratory is supported by the US Department of Energy, under contract $W-7405-E N G-36$.

[1] J. Jäckle, Rep. Prog. Phys. 49, 171 (1986).

[2] K.L. Ngai, J. Non-Cryst. Solids 275, 7 (2000).

[3] P. Lunkenheimer, U. Schneider, R. Brand, and A. Loidl, Contemporary Physics 41, 15 (2000).

[4] A. Ansari et al., Biophysical Chemistry 26, 337 (1987).

[5] I.E.T. Iben et al., Phys. Rev. Lett. 62, 1916 (1989).

[6] T.A. Jackson, M. Lim, and P.A. Anfinrud, Chem. Phys. 180, 131 (1994).

[7] K. Fritsch, A. Eicker, J. Friedrich, B.M. Kharlamov, and J.M. Vanderkooi, Europhys. Lett. 41, 339 (1998).

[8] A. Xie, L. van der Meer, W. Hoff, and R.H. Austin, Phys. Rev. Lett. 84, 5435 (2000).

[9] J. Bredenbeck, J. Helbing, J.R. Kumita, G.A. Woolley, and P. Hamm, Proc. Natl. Acad. Sci. USA 102, 2379 (2005).

[10] H. Frauenfelder, S.G. Sligar, and P.G. Wolynes, Science 254, 1598 (1991).

[11] E.B. Brauns, M.L. Madaras, R.S. Coleman, C.J. Murphy, and M.A. Berg, Phys. Rev. Lett. 88, 158101 (2002).

[12] G. Altan-Bonnet, A. Libchaber, and O. Krichevsky, Phys. Rev. Lett. 90, 138101 (2003).

[13] T. Dauxois, M. Peyrard, and A.R. Bishop, Phys. Rev. E 47, 44 (1993).

[14] A. Campa and A. Giansanti, Phys. Rev. E 58, 3585 (1998).

[15] C.H. Choi, G. Kalosakas, K.Ø. Rasmussen, M. Hiromura, A. Bishop, and A. Usheva, Nucleic Acids Res. 32, 1584 (2004).

[16] S. Ares, N.K. Voulgarakis, K.Ø. Rasmussen, and A.R. Bishop, Phys. Rev. Lett. 94, 035504 (2005).

[17] T. Dauxois and M. Peyrard, Phys. Rev. E 51, 4027 (1995). 
[18] N. Theodorakopoulos, T. Dauxois, and M. Peyrard, Phys. Rev. Lett. 85, 6 (2000).

[19] G. Kalosakas, K.Ø. Rasmussen, A. Bishop, C.H. Choi, and A. Usheva, Europhys. Lett. 68, 127 (2004).

[20] P.W. Fenimore, H. Frauenfelder, B.H. McMahon, and R.D. Young, Proc. Natl. Acad. Sci. USA 101, 14408 (2004).

[21] G. Kalosakas, K.Ø. Rasmussen, and A.R. Bishop, J. Chem. Phys. 118, 3731 (2003); Synth. Met. 141, 93 (2004).

[22] G. Kalosakas, K.L. Ngai, and S. Flach, Phys. Rev. E 71, 061901 (2005).

[23] N.K. Voulgarakis, G. Kalosakas, K.Ø. Rasmussen, and A.R. Bishop, Nano Lett. 4, 629 (2004).

[24] K.L. Ngai, R.W. Rendell, and H. Jain, Phys. Rev. B 30, 2133 (1984).

[25] C. León, M.L. Lucia, and J. Santamaria, Phys. Rev. B 